\title{
Durchflußzytometrische nichtradioaktive Methode zur Bestimmung der zytotoxischen Aktivität von natürlichen Killerzellen: Vergleich mit dem klassischen ${ }^{51}$ Cr-Release-Assay
}

\author{
Flow Cytometric Non-radioactive Method for the Determination of the Cytotoxic Activity of \\ Natural Killer Cells: Comparison with the Classical ${ }^{51} \mathrm{Cr}$-Release Assay
}

\author{
H. Lötzerich ${ }^{1,2}$, W. Hirt ${ }^{3}$
}

Zusammenfassung: Die Aktivität von natürlichen Killer(NK)-Zellen im Blut ist ein wichtiger Parameter des Immunstatus. Ihre Bestimmung kann bei der Diagnose, Prognose und bei der Überwachung des Therapieverlaufs verschiedener Erkrankungen hilfreich sein. Dazu gehören Krebserkrankungen, Multiple Sklerose, systemischer Lupus erythematodes, das Sjögren Syndrom, das Chronique Fatigue Syndrom und die HIVInfektion. In der immunologischen Forschung wird zur Bestimmung der zytotoxizität von NK-Zellen der Chrom-Release-Test als Standard-Methode eingesetzt, wobei die Lyserate der NK-Zellen mit Hilfe radioaktiv markierter Tumorzellen (K562) quantifiziert wird. In der vorliegenden Arbeit werden zwei Methoden zur Bestimmung der NK-Zellaktivität gegenübergestellt. Dazu wurden gleichzeitig 25 . Proben mit einem Chrom-Release-Test und einem nichtradioaktiven durchflußzytometrischen Testverfahren unter Verwendung von Fluoreszenzfarbstoffen zur Unterscheidung vitaler und toter Targetzellen untersucht. Die mit beiden Methoden erhaltenen Ergebnisse weisen keine signifikanten Unterschiede $(p<0,05)$ auf. Zur Vermeidung von Radioaktivität ist deswegen das neue durchflußzytometrische Verfahren die Methode der Wahl, zumal es insgesamt einen wesentlich geringeren Zeitaufwand erfordert.

Schlüsselwörter: Kïllerzellen, Natürliche/Blut; Zytotoxizität, Immunologische/Blut; Chrom Radioisotope; Durchflußzytometrie; Carbocyanine; Fluoreszenzfarbstoffe.

\footnotetext{
${ }^{1}$ Deutsche Sporthochschule Köln, Institut f. Experimentelle Morphologie, Lehrstuhl für Morphologie und Tumorforschung

2 Korrespondenzadresse: Priv.-Doz. Dr. Helmut Lötzerich, Deutsche Sporthochschule Köln, Institut f. Experimentelle Morphologie, Lehrstuhl für Morphologie und Tumorforschung, D-50927 Köln. Fax: +49-221-4995765

3 Orpegen Pharma, Heidelberg
}

Eingegangen: 18. Juni 1996/Angenommen: 11. November 1996
Summary: The activity of natural killer (NK) cells in peripheral blood is an important parameter of the immune status. Activity determination can be used for diagnosis, prognosis and monitoring of therapy under different disease states like cancer, multiple sclerosis, systemic lupus erythematosus, Sjogren's syndrome, chronic fatigue syndrome and infection with human immunodeficiency virus. In immunological research the chromium release assay is the standard method for the determination of the cytotoxicity of NK cells. In this method the lysis of ${ }^{51}$ chromium-labelled tumor cells (K562) is observed. In the present investigation two methods for measuring the activity of NK cells were compared. NK cell activity was simultaneously determined in 25 blood samples with both a ${ }^{51}$ chromium release assay and a flow cytometric procedure using fluorescent dyes for the differentiation between vital and killed target cells. Results obtained with both methods did not show significant differences $(p<$ 0.05). As the flow cytometric method does not require radioactive substances and as it is in total less timeconsuming, it appears to be the method of choice.

Keywords: Killer Cells, Natural/blood; Cytotoxicity, Immunologic/blood; Chromium Radioisotopes; Flow Cytometry; Carbocyanines; Fluorescent Dyes.

D ie natürlichen Killer (NK)-Zellen gehören neben den Phagozyten zu den zellulären Bestandteilen des unspezifischen Abwehrsystems. Beim Menschen ist der größte Teil der NK-Zellen morphologisch anhand intrazytoplasmatischer azurophiler Granula zu erkennen, weshalb die Zellen.in der Literatur auch als LGLs (large granular lymphocytes) bezeichnet werden. Als Effektorzellen der natürlichen Immunität verfügen sie nicht über ein immunologisches Gedächtnis

Nicht standardisierte Abkürzungen: $C D$, cluster designation; $\mathrm{cpm}$, counts per minute; HIV, human immunodeficiency virus: LGL, large granular lymphocyte; NK, natural killer (cell); PBMC. peripheral blood mononuclear cell; PBS, phosphate-buffered saline; RPMI, Roswell Park Memorial Institute (medium); RT, Raumtemperatur. 
und untcrliegen nicht der MHC-Restriktion. Sie sind in der Lage. ihre zytotoxische Wirkung ohne vorherige Stimulation 70 entfalten. Als akzessorische Effekıorzellen miı Fc-Rezeptor können sie verschiedene immunologisch bedeutende Mediatoren, u.a. Interferon- $\gamma$, sezernieren. Die NK-Zcllen werden in der Regel anhand ihrer Oberflächenmarker CD16 und CD56 identifiziert. Dabei handelt es sich um den Fc RIII (CD 16), der von mehr als $95 \%$ der Zellen exprimiert wird, und das NCAM (neural cell adhesion molecule: CD56), das $80 \%$ bis $90 \%$ der NK-Zellen tragen [1-3]. Mit Hilfe der Durchflußzytometrie lassen sich damit die NK-Zellen als CD3- und CD16/CD56+ darsiellen. Weitere für NK-Zellen charakteristische Oberfliichenmarker sind aus der Tabelle $1[1,4,2] \mathrm{zu}$ entnehmen.

Tabelle 1 Die wichtigsten Oberflächenmarker von NKZellen (nach $[1,2,4]$ )

\begin{tabular}{lll}
\hline $\begin{array}{l}\text { Oberflächen- } \\
\text { marker }\end{array}$ & Merkmal & $\begin{array}{l}\text { Vertei- } \\
\text { lung }\end{array}$ \\
\hline CD 56 & Neural Cell Adhesion Molecule & $>95 \%$ \\
CD 16 & FC $\gamma$ R III & $80-90 \%$ \\
CD $11 b$ & Komplementrezeptor (CR 3) & $80-90 \%$ \\
CR 1 & Komplementrezeptor (CR 1) & $80-90 \%$ \\
CD 7 & FC $\mu$ R & $80-90 \%$ \\
\hline
\end{tabular}

Die biologische Bedeutung der NK-Zellen liegt in der Abwehr von Viren und Tumorzellen [5,6]. Im Rahmen der Krebsabwehr spielen sie eine große Rolle bei der Abstoßung transplantierter Tumor- oder Knochenmarkszellen sowie der Prävention von Metastasen. Dagegen verlieren sie an immunologischer Kompetenz bei bereits etabliertem Krebs, wobei ihre Zahl abnimmt und ihre Funktion eingeschränkt ist [7]. Ihre antitumorale Wirkung liegt damit mehr in der frühen Phase im Kàmpf gegen einen entstehenden Tumor. Dies erklärt auch die reduzierte NK-Zellaktivität von Krebspatienten bei der Diagnose und in der ersten Phase der Rehabilitation neben einer möglichen Suppression als Folge verschiedener Krebstherapien [2]. Eine erniedrigte NK-Zellaktivität ist häufig ein Zeichen für eine Immunschwäche. So ist eine Abnahme der NK-Zellzahl und -funktion im Laufe einer HIV-Infektion zu beobachten $[2,8,9]$. Eine Abnahme der NKZellzytotoxizität spielt auch bei anderen Erkrankungen wie der Multiplen Sklerose [10], beim systemischen Lupus erythematodes [11], dem Sjögren Syndrom $[12,13,14]$ und dem Chronique Fatigue Syndrom [15] eine Rolle. Auch bei starken psychischen Belastungen oder Depressionen kann eine reduzierte NK-Zellaktivität nachgewiesen werden $[16,17,18,19,20]$.

Daher ist die Bestimmung der NK-Zellzytotoxizität ein wichtiger Parameter bei der Ermittlung eines Immunstatus. Sie kann hilfreich bei der Diagnose, Prognose und im Therapieverlauf sein [21]. In der immunologischen Forschung wird zur Bestimmung der Zytotoxizität von NK-Zellen der ${ }^{51}$ Chrom-Release-Test als Standard-Methode eingesetzt, wobei die Lyserate der NK-Zellen mit Hilfe radioaktiv-markierter Tumorzellen (K562) quantifiziert wird. Ziel dieser Arbeit war die Erprobung eines modifizierten Testverfahrens, das auf den Einsatz der Radioaktivität zu Gunsten eines Fluoreszenz-Signals zu verzichten erlaubt. Die Ergebnisse beider Testverfahren wurden gleichzeitig erhoben und miteinander verglichen.

\section{Material und Methoden}

\section{Probanden}

Zur Bestimmung der NK-Zellwerte wurden 25 venöse Blutproben von gesunden Probanden morgens zwischen 8 und 9 Uhr im nüchternen Zustand liegend aus der Cubitalvene entnommen. Davon wurden $2,7 \mathrm{ml}$ EDTA-Blut für die Erstellung des Blutbildes und die durchflußzytometrische Immunphänotypisierung sowie $5 \mathrm{ml}$ Ammoniumheparinat-Blut zur Bestimmung der NK-Zell-Lyse verwendet.

\section{Durchflußzytometrische Analyse zur Bestimmung der NK-Zellzahl}

Der prozentuale Anteil der NK-Zellen wurde mit Hilfe eines Durchflußzytometers bestimmt. Dazu wurden $100 \mu 1$ EDTA-Vollblut mit $10 \mu \mathrm{l}$ einer Mischung aus Fluoreszenz-markierten monoklonalen Antikörpern (CD56/CD16; Becton Dickinson GmbH, Heidelberg) 15 min bei Raumtemperatur (RT) inkubiert. Danach wurden die Ansätze in einem Probenvorbereitungssystem (Q-Prep, Coulter Electronics GmbH, Krefeld) weiterverarbeitet, wobei die Erythrozyten lysiert werden und eine Fixierung der Leukozyten erfolgt. Die durchflußzytometrische Bestimmung wurde innerhalb einer Stunde am Epics Profile II (Argonlaser $488 \mathrm{~nm}$; Coulter, Krefeld) durchgeführt.

Die absoluten Zahlen der NK-Zellen wurden mit Hilfe der Leukozyten- und Lymphozytenzahlen errechnet. Die Bestimmung der Gesamtleukozyten und der prozentualen Differenzierung in Lymphozyten, Monozyten und der Granulozyten erfolgte an einem Leukozytenzählgerät (MD-18, Coulter) aus EDTAVollblut.

\section{Bestimmung der NK-Zellzytotoxizität :}

Die nichtradioaktive durchflußzytometrische Bestimmung der NK-Zellzytotoxizität erfolgte mit kryokonservierten Targetzellen und weiteren Reagenzien eines kommerziell erhältlichen Testkits (NKTEST ${ }^{\circledR}$ der Fa. ORPEGEN Pharma, Heidelberg).

\section{Isolierung der Effektorzellen}

Periphere mononukleäre Blutzellen (PBMC) wurden nach der Standard-Ficoll-Hypaque (Dichte 1,077 ) Methode gewonnen. Dazu wurden 5 ml Heparin-Blut im Verhältnis 1:2 mit Phosphat-gepufferter Kochsalzlösung (PBS) verdünnt und vorsichtig in ein mit $5 \mathrm{ml} \mathrm{Fi-}$ coll gefülltes Zentrifugenröhrchen gegeben. AnschlieBend wurde bei RT 20 min mit $700 \mathrm{~g}$ (ohne Bremse) 

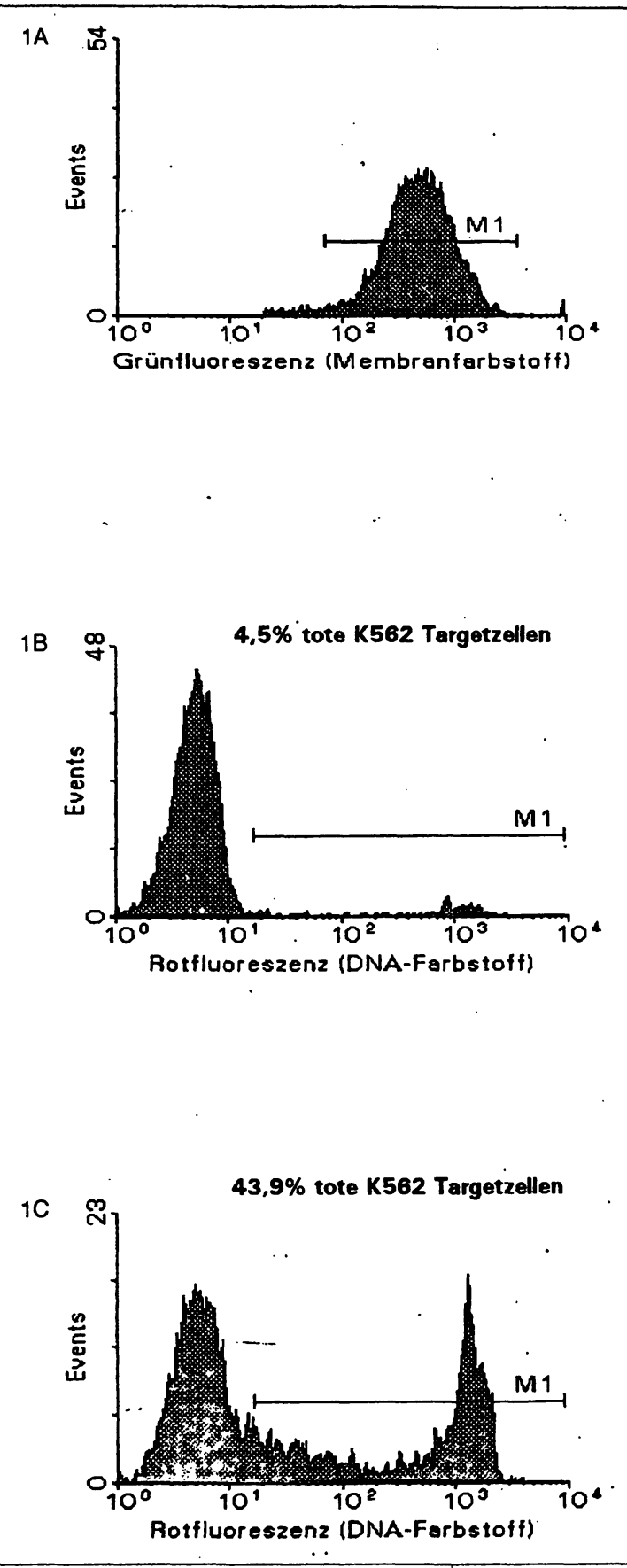

Abbildung 1 Bestimmung der NK-Zellcytotoxicität mittels Durchflußzytometrie.

Oben:Setzen eines „Gate" oder „Bitmap" auf grünfluoreszente Targetzellen im FL1-Histogramm.

Mitte:Typisches FL2-Histogramm zur Bestimmung des Anteils an rotfluoreszenten, toten Targetzellen im Kontrollansatz (Targetzellen ohne Effektorzellen)

Unten: Typisches FL2-Histogramm zur Bestimmung des Anteils an rotfluoreszenten, toten Targetzellen im Testansatz (Target- und Effektorzellen, Effektor : Targetverhăltnis von $50: 1$ ). zentrifugiert. Die obere Schicht wurde entfernt und die Interphase, die die mononukleären Zellen beinhaltet, vorsichtig mit Hilfe einer Pipette abgenommen. Diese Zellsuspension wurde mit PBS gemischt und bei RT $10 \mathrm{~min}$ mit $250 \mathrm{~g}$ zentrifugiert. Nach nochmaligem Waschen mit PBS wurden die Zellen auf die Konzentrationen $5 \times 10^{6} / \mathrm{ml}, 2,5 \times 10^{6} / \mathrm{ml}$ und $1,25 \times 10^{6} / \mathrm{ml}$ mit Komplettmedium (RPMI 1640 Medium $+10 \%$ (v/v) fötales Rinderserum) eingestellt.

\section{Präparation der Targetzellen}

Die bei $-70{ }^{\circ} \mathrm{C}$ gelagerten $\mathrm{K} 562$ Zellen wurden schnell, innerhalb von $2 \mathrm{~min}$, im Wasserbad bei $37^{\circ} \mathrm{C}$ aufgetaut und sofort in ein mit $50 \mathrm{ml}$ vorgewärmtem Komplettmedium gefülltes Zentrifugenröhrchen überführt. Nach kurzem Schwenken wurde bei RT $5 \mathrm{~min}$ mit $120 \mathrm{~g}$ zentrifugiert. Der Überstand wurde entfernt und mit $10 \mathrm{ml}$ Komplettmedium resuspendiert. Die Targetzellen wurden zum einen mit dem grünfluoreszenten Membranfarbstoff $\left(\mathrm{DiOC}_{18}\right)$ und zum anderen mit $\mathrm{Na}_{2}{ }^{51} \mathrm{CrO}_{4}$ markiert.

Markierung der Targetzellen mit dem Membranfarbstoff (DiOC 18$)$

$\mathrm{Zu} 5 \mathrm{ml}$ der s.o. präparierten Zellsuspension wurden $20 \mu \mathrm{l}$ Membranfarbstoff $\mathrm{DiOC}_{18}$ gegeben und nach sofortigem kurzen Mischen für $15 \mathrm{~min}$ bei $37^{\circ} \mathrm{C}$ im Wasserbad inkubiert. Anschließend wurden $10 \mathrm{ml}$ Komplettmedium zugegeben und bei RT $5 \mathrm{~min}$ mit $120 \mathrm{~g}$ zentrifugiert. Danach wurden die Zellen auf eine Konzentration von $1 \times 10^{5} / \mathrm{ml}$ mit Komplettmedium eingestellt.

Dieser Schritt entfällt bei Einsatz des kommerziell erhältlichen Testkits, da dieser Targetzellen enthält, die bereits mit dem Membranfarbstoff markiert sind.

\section{Markierung der Targetzellen mit $\mathrm{Na}_{2}{ }^{51} \mathrm{CrO}_{4}$}

Die s.o. präparierte Zellsuspension wurde auf $1 \mathrm{x}$ $10^{6} / 0,5 \mathrm{ml}$ Komplettmedium eingestellt. Nach Zugabe von $100 \mathrm{Ci} \mathrm{Na}_{2}^{51} \mathrm{CrO}_{4}$ (spezifische Aktivität von 400 bis $1200 \mathrm{mCi} / \mathrm{mg}$; Fa. Amersham) wurden die Zellen $60 \mathrm{~min}$ bei $37{ }^{\circ} \mathrm{C}$ im Brutschrank inkubiert. Anschließend wurde die Zellsuspension bei RT 8 min mit $400 \mathrm{~g}$ zentrifugiert und mit Komplettmedium resuspendiert. Dieser Waschvorgang wurde noch zweimal wiederholt. Danach wurden die Zellen auf $1 \times 10^{5} / \mathrm{ml}$ mit Komplettmedium eingestellt.

\section{Testansatz der nichtradioaktiven Methode}

Je $100 \mu \mathrm{l}$ Targetzellen und $100 \mu \mathrm{l}$ Effektorzellen der jeweiligen Zellkonzentration $(50: 1 ; 25: 1 ; 12,5: 1)$ wurden in einem Zentrifugenröhrchen zusammengegeben und bei RT 3 min mit $120 \mathrm{~g}$ zentrifugiert. Als Kontrolle diente ein Ansatz ohne Zugabe von Effektorzellen, um die spontane Absterberate der Targetzellen zu bestimmen. Die Röhrchen wurden mit Deckel versehen und $4 \mathrm{~h}$ bei $37^{\circ} \mathrm{C}$ im Wasserbad inkubiert. Nach dieser Inkubationszeit wurden die Proben in ein Eiswasserbad gestellt und mit je $50 \mu \mathrm{l}$ DNA-Färbelösung versetzt und auf dem Vortexer gemischt. Der rot- 
fluoreszentc DNA-Farbstoff färbt dic durch die Aktivitäı der NK-Zcllen membrangeschädigten Targetzellen an. Die Proben wurden nach 3-5 min mil dem Durchflußzytometer (Epics Profile II, Coulter. Krefeld) analysiert. Dabei wurde ein Prohenvolumen von $180 \mu 1$ bei einer DurchfluBrate von $60 \mu \mathrm{l} / \mathrm{min}$ untersucht. Durch das Setzen eines "Gate“ oder .Bitmap" wurden zur Unterscheidung zwischen Effektor- und Targetzellen nur grïnfluoreszente Targetzellen (s. Abb. 1A) registriert. Ausgewertet wurde der Anteil an toten, d.h. rotfluoreszenten Targetzellen (s. Abb. IB. IC). Die prozentuale Lyserate wurde durch die Differenz von Kontrollwert (Targetzellen ohne Effektoren) und Tesiwert (Targetzellen mit Effektoren) ermittelt.

\section{Ansatz des radioaktiven Chrom-Release-Tests}

Im Dreifach-Ansalz wurde eine Mikrotiterplatte mit je $100 \mu l$ Targetzellen und $100 \mu$ l Effektorzellen der jeweiligen Zellkonzentration $(50: 1 ; 25: 1 ; 12,5: 1)$ beschickt und bei RT 3 min mit $25 \mathrm{~g}$ zentrifugiert. Proben zur Ermittlung der spontanen (Targetzellen ohne Zugabe von Effektorzellen) und maximalen IsotopFreisetzung (Targetzellen mit Zugabe von 0,25\% Dodecyl Schwefelsäure Natriumsalz = SDS) wurden parallel angesetzt. Die Mikrotiterplatte wurde $4 \mathrm{~h}$ bei $37^{\circ} \mathrm{C}$ im $\mathrm{CO}_{2}$-Brutschrank inkubiert. Nach dieser Zeit wurde bei RT 5 min mit $400 \mathrm{~g}$ zentrifugiert und aus allen Proben $100 \mu \mathrm{l}$ zellfreier Überstand entnommen.
Die enthaltene Radioaktivität (cpm) wurde in einem Gammacounter (Fa. Berthold) gemessen. Die Lyserate in Prozent wurde nach folgender Formel errechnet:

Lyserate $=\frac{(\mathrm{cpm} \text { der Probe } \cdot \mathrm{cpm} \text { Spontanfreisetzung }) \times 100}{\mathrm{cpm} \text { Maximalfreisetzung }}$

\section{Berechnung der NK-Zellaktivität pro Zelle}

Die Berechnung der NK-Zellaktivität pro Zelle erfolgte über Lytic Units nach der Formel von Pross und Mitarbeitern [22]:

NK-Zellaktivität pro Zelle $=$

Lytic Units

$\overline{\% \text { NK-Zellen x (106 PBMC - \%.Monozyten })}$

Dabei wird ein „Lytic Unit" definiert als Zahl der Effektorzellen, die benötigt werden, um $20 \%$ von 10.000 Targetzellen zu lysieren $[22,23]$.

Berechnung der NK-Zellen zur Lyse einer Targetzelle Zur Berechnung der NK-Zellen, die zur Lyse einer Targetzelle benötigt werden, wird die absolute Zahl der Gesamtleukozyten, der prozentuale Anteil Lymphozyten und der NK-Zellen benötigt, um dann die absolute Zahl der NK-Zellen im Zellansatz zu ermitteln. Anhand der Lyserate wird dann berechnet, wieviele NK-Zellen nötig sind, um eine K562-Zelle zu töten.

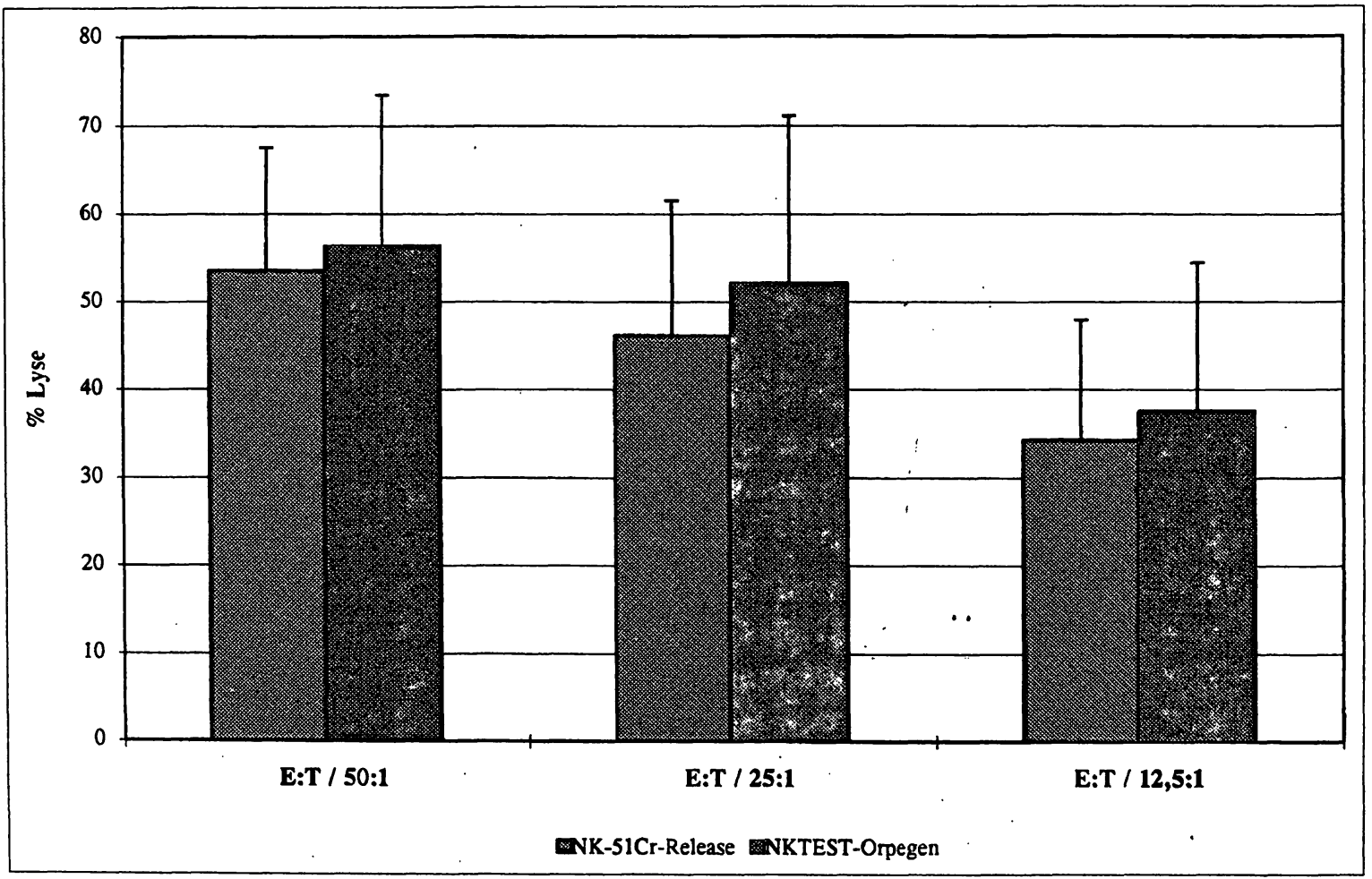

Abbildung 2 Vergleich der Lyserate im ${ }^{51}$ Chrom-Release-Test zur nichtradioaktiven durchflußzytometrischen Methode $(n=25, p>0,05)$ 


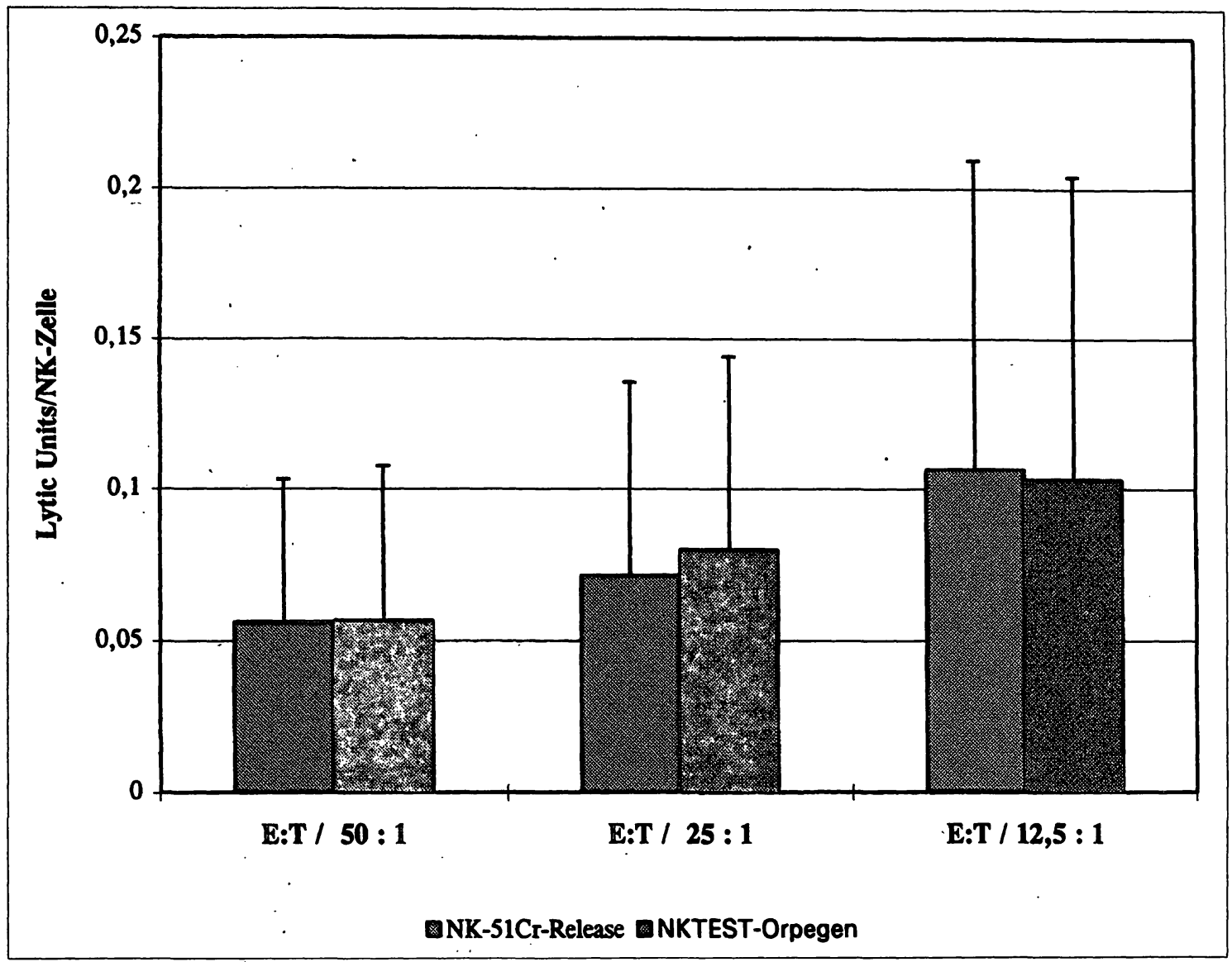

Abbildung 3 Vergleich der Lytic Units pro NK-Zelle im ${ }^{51}$ Chrom-Release-Test zur nichtradioaktiven durchflußzytometrischen Methode $(n=25, p>0,05)$

\section{Statistik}

Die statistische Auswertung wurde an einem Personalcomputer mit Hilfe des Programms SPSS/PC+ (Version 4.0) durchgeführt. Zum Vergleich der Stichproben wurde ein t-Test eingesetzt, wobei das Signifikanzniveau auf $p<0,05$ festgelegt wurde.

\section{Ergebnisse}

Die Ergebnisse der beiden unterschiedlichen Methoden weisen bei den gewählten Effektor : Targetver-. hältnissen von $50: 1,25: 1$ und 12,5:1 keine signifikanten Unterschiede bei der Lyserate auf (Abb. 2). Die Werte der nichtradioaktiven Methode liegen im Mittelwert bei allen Effektor : Targetverhältnissen immer etwas höher, was aber statistisch keine Bedeutung hat. Dementsprechend treten auch keine statistischen Unterschiede nach der Berechnung über die Lytic Units auf (Abb. 3). Ebenso liefern beide Methoden vergleichbare Ergebnisse, wenn die Zahl der NK-Zellen berechnet wird, die erforderlich sind, um eine Targetzelle zu lysieren (Abb. 4). In Abb. 5 sind beispielhaft für ein Effektor : Targetverhältnis von $25: 1$ die Lyseraten der durchflußzytometrischen Methode als $\mathrm{x}$ Werte dargestellt, die $y$-Werte repräsentieren die Werte des ${ }^{51} \mathrm{Cr}$-Release-Tests. Wie ersichtlich, besteht eine sehr gute Korrelation zwischen beiden Methoden, der Korrelationskoeffizient beträgt 0,935 .

Weiterhin wurde die Präzision der durchflußzytometrischen Methode anhand einer dreifachen Bestimmung der NK-Zellzytotoxizität von PBMC-Proben gesunder Probanden ermittelt. Wie Tabelle 2 entnommen werden kann, ergaben sich durchschnittliche Variationskoeffizienten von 5,9 bis $7,6 \%$.

\section{Diskussion}

Die Befunde zeigen deutlich; daß die beiden Methodenalternativ benutzt werden können. Da sich die Ergebnisse nicht signifikant unterscheiden, ist die nichtradioaktive Methode als Methode der Wahl zu empfehlen. Damit wurde weiterhin gezeigt, daß die Markierung der Effektorzellen mit dem Membranfarbstoff zu gleichen Resultaten führt wie die radioaktive Markie- 


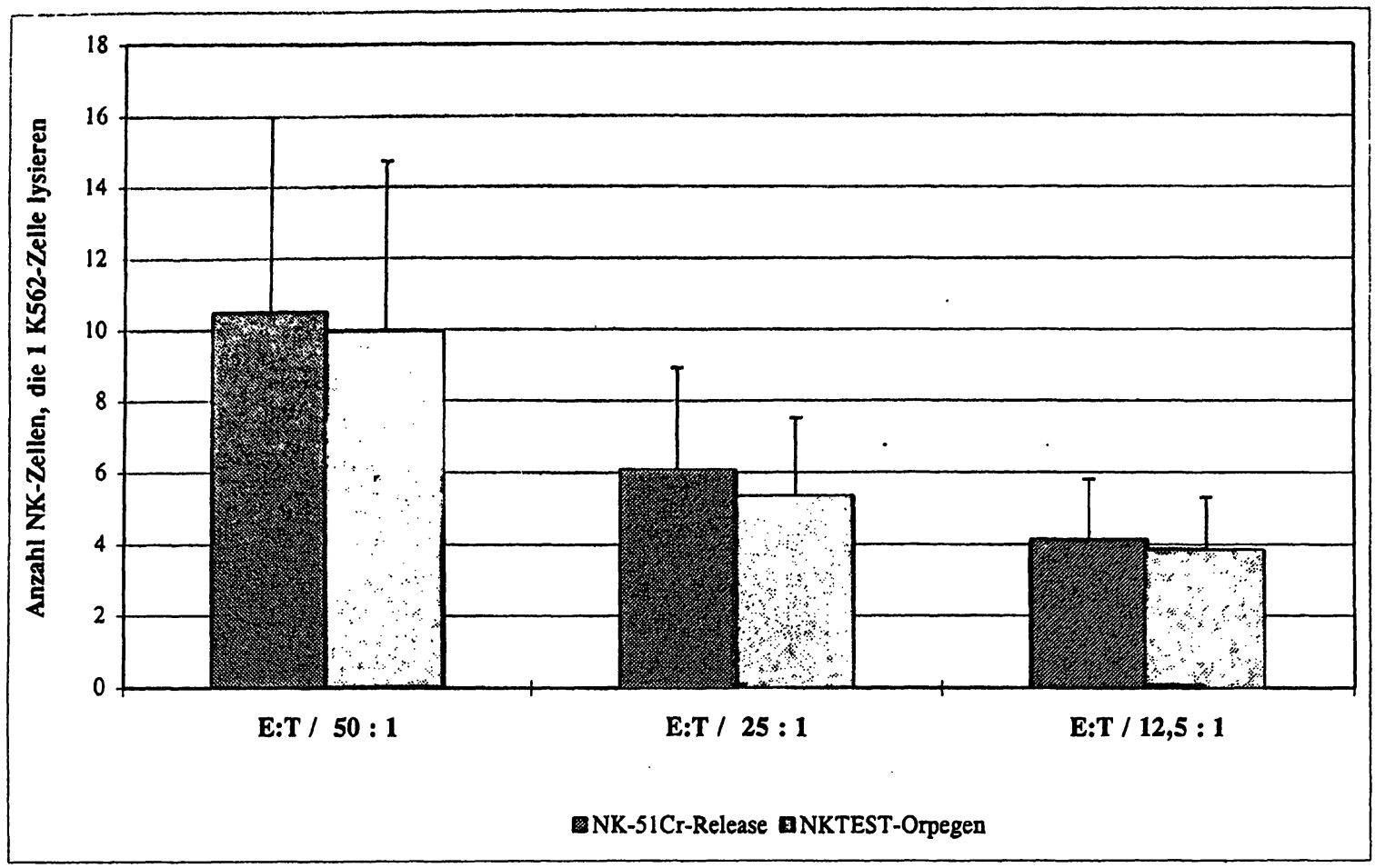

Abbildung 4 Vergleich der NK-Zellaktivität pro Targetzelle im ${ }^{51}$ Chrom-Release-Test zur nichtradioaktiven durchflußzytometrischen Methode $(n=25, p>0,05)$

rung mit ${ }^{51}$ Chrom und daß die Art der Markierung keinen Einfluß auf die Effektorzellen oder deren Lysierbarkeit hat. Ebenso entstehen keine Unterschiede durch die unterschiedlichen Meßprinzipien (Radioaktivitätmessung oder Durchflußzytometrie).

Die in der Literatur gefundenen unterschiedlichen Werte für Lyseraten des Chrom-Release-Tests lassen sich durch unterschiedliche Effektor:Targetverhältnisse erklären. Dabei spielt nicht nur das Verhältnis, sondern auch die Zusammensetzung der Effektorzellen eine große Rolle. Geht man davon aus, daß nur die NK-Zellen für die Lyse der K562 Targetzellen verantwortlich sind, spielt der Anteil der NK-Zellen in der Effektorzellpopulation eine wichtige Rolle. In der Regel werden PBMC ungereinigt als Effektorzellen verwendet, d.h. es handelt sich hier um eine Mischpopulation von Monozyten und Lymphozyten. Über die
Eliminierung der Monozyten, wobei deren Adhärenzverhalten ausgenutzt wird, kann der Anteil der NKZellen erhöht werden. Insbesondere Monozyten können über die Produktion von Prostaglandinen die NKZellen in ihrer Funktion beeinträchtigen (24). Dementsprechend werden mit zunehmendem Anteil an NKZellen in der Effektorzellpopulation höhere Lyseraten erzielt. Die Reinigung kann bis zur Isolierung von NK-Zellen nach der Methode von Cosentino und Cathart [25] führen. Der Nachteil liegt aber in einer sehr zeit- und materialaufwendigen Arbeit, die in der Routine nicht $\mathrm{zu}$ realisieren ist; darüber hinaus werden größere Blutmengen benötigt.

Während die Lyserate über die natürliche Killeraktivität des peripheren Blutes eine Auskunft gibt, wird versucht durch die nachträgliche Berechnung des genauen Effektor:Targetverhältnisses eine Aussage über

Tabelle 2 Präzision der durchflußzytometrischen Methode

\begin{tabular}{|c|c|c|c|}
\hline & Lyserate $(E: T=12,5: 1)$ & Lyserate $(E: T=25: 1)$ & Lyserate $(E: T=50: 1)$ \\
\hline $\begin{array}{l}\text { Wertebereich } \\
\text { Durchschnittlicher Variationskoeffizient (\%) } \\
n\end{array}$ & $\begin{array}{l}7,8-33,5 \\
6,9 \\
6\end{array}$ & $\begin{array}{l}15,8-54,1 \\
7,6 \\
6\end{array}$ & $\begin{array}{l}20,4-63,7 \\
5,9 \\
6\end{array}$ \\
\hline E:Effektorzellen, T:Targetzellen & $\cdot$ & & \\
\hline
\end{tabular}




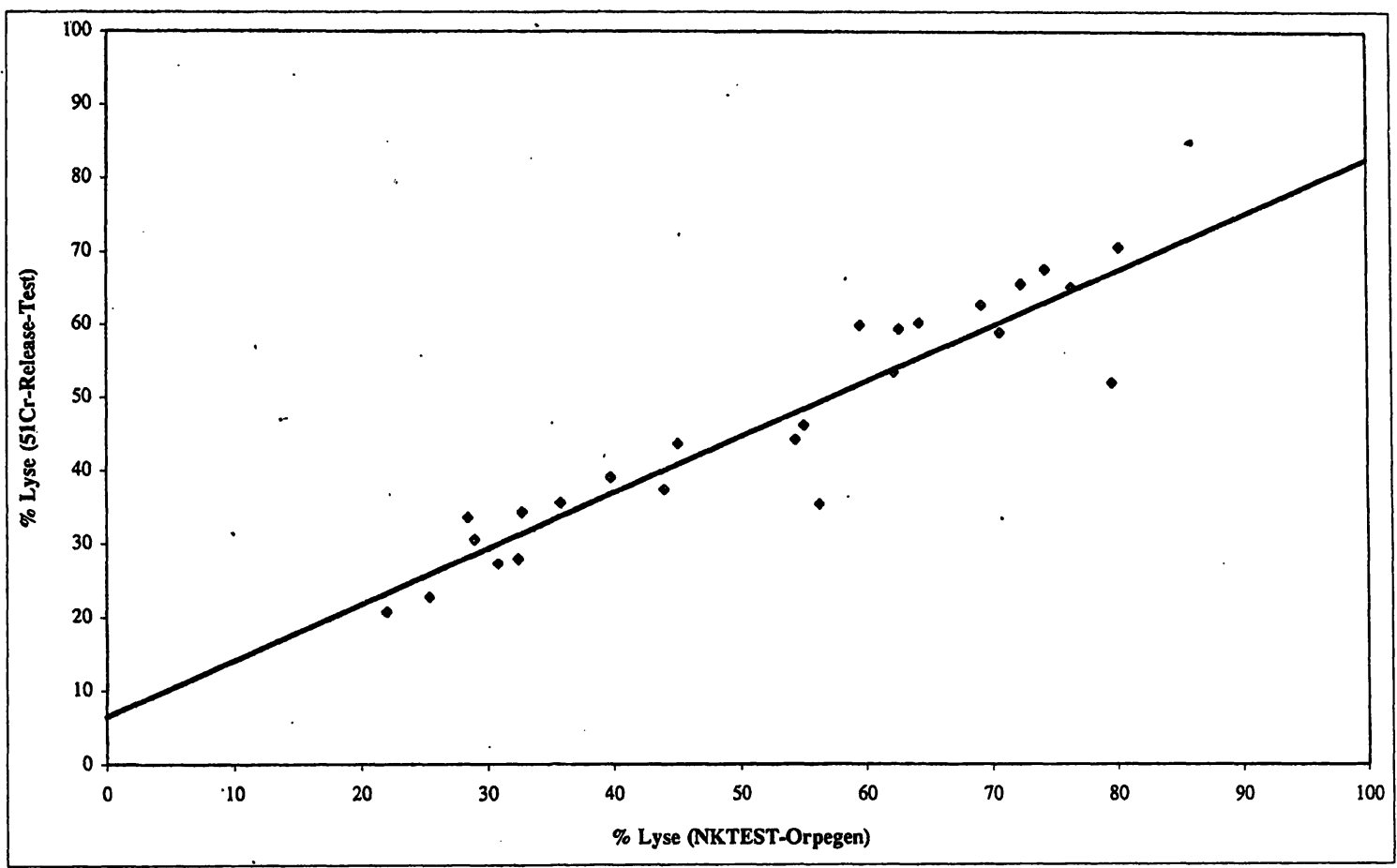

Abbildung 5 Korrelation zwischen ${ }^{51}$ Chrom-Release-Test und durchflußzytometrischer Methode. Die Lyseraten der durchflußzytometrischen Methode sind auf der $x$-Achse aufgetragen, die Lyseraten des ${ }^{51}$ Chrom-Release-Tests auf der $y$-Achse. Dargestellt sind individuelle Werte von 25 Vergleichsmessungen bei einem Effektor : Targetverhältnis von $25: 1$, Korrelationskoeffizient $=0,935$, Steigung $=0,76$.

die Lyseaktivität der einzelnen NK-Zellen zu machen. Da die beiden Testmethoden sich bei der Lyserate nicht unterschieden, ist es leicht verständlich, daß die Berechnungen über die Lytic Units oder die Menge der NK-Zellen, die benötigt werden, um eine Targetzelle zu lysieren, ebenfalls zu keinen Unterschieden in den Ergebnissen beider Tests führten. Da die Effektor:Targetverhältnisse und auch die Zellzahlen in der Literatur nicht immer standardisiert sind, erscheint die Berechnung pro NK-Zelle geeigneter als die Berechnung über die Lytic Units, obwohl beide Berechnungen letztendlich zum gleichen Ergebnis führen.

Für die Durchführung in der Praxis ist es wichtig, die benötigte Blutmenge zu minimieren, um die Belastung der Patienten besonders bei Verlaufsbeobachtungen so gering wie möglich zu halten. Weiterhin ist eine möglichst schnelle Verarbeitung der Blutproben anzustreben. Die gesamte Testdauer sollte so kurz sein, daß der Test in der Routine auch mit größeren Probenzahlen durchführbar ist. Hier hat sich der alternative nichtradioaktive Test ebenfalls bewährt, da er schon nach zwei Stunden und nicht erst nach vier Stunden, wie beim Chrom-Release-Test, reproduzierbare Ergebnisse liefert. Außerdem entfällt bei dem kommerziell erhältlichen Testkit die mühsame und zeitaufwendige Anzucht der K562 Targetzellen, da im Kit kryokonservierte fluoreszenzmarkierte Zellen enthalten sind.

Der vermehrte Einsatz von NK-Zelltesten wird in der Zukunft zeigen welche Bedeutung die Aktivität der
NK-Zellen bei der Diagnose und der Überwachung des Therapieverlaufs verschiedener Erkrankungen hat.

\section{Danksagung}

Unser Dank gilt Magdalena Euler, Margot Maskus, Alexander Wink und Hannes Höchemer für ihre exzellente technische Mitarbeit und der großen Geduld bei der Erstellung des Manuskripts.

\section{Literaturverzeichnis}

1. Robertson MJ, Ritz J. Biological and clinical relevance of human natural killer cells. Blood 1990;76:2421-38.

2. Roitt I, Brostoff J, Male D. Kurzes Lehrbuch der Immunologie. Stuttgart (DE): Thieme Verlag, 1991.

3. Janeway C, Travers P. Immunobiologie. Heidelberg(DE):Spektrum Akademischer Verlag, 1995.

4. Landey A, George S, Bray R. Phenotyping Cells of the Immune System: Methodes and Applicatiōns. In: Coon JS, Weinstein RS, eitors. Diagnostic flow cytometry. New York: United States and Canadian Academy of Pathology Inc., 1991

5. Whiteside TL, Herbermann, RB. Characteristics of natural killer cells and lymphocyte-activated killer cells. Immunol Allergy Clin Am 1990;10: 663-704.

6. Trinchieri G. Biology of natural killer cells. Adv Immunol 1989;47:187-205.

7. Kadish AS, Doyle AT, Steinhauer EH, Ghossein NA. Natural cytotoxicity and interferon production in human cancer: Deficient natural killer activity and normal interferon production in patients with advanced disease. J Immunol 1981;127:1817. 
8. Rosenberg ZF. Fauci AS. The immunopathogenesis of HIV infection. Adv Immunol 1989:47:377-431.

9. Brostoff J, Scadding GK. Male D, Roitt IM. Klinische Immunologie. Weinhein(DE): VCH Verlagsgesellschaft, 1993.

10. McGarry RC, Roder JC, Brunet D. Mechanisms of natural killer cell depression in multiple sclerosis. In:Herberman RB, editor. NK Cells and other Natural Effektor Cells. New York (USA): Academic Press. 1982:1219-25.

11. Strannegard $O$, Hermodsson $S$, Westberg $G$. Interferon and natural killer cells in systematic lupus erythematosus. Clin Exp Immunol 1982;50:246.

12. Goto M. Tanimoto K. Horuichi Y. Natural killer cell-mediated cytotoxity in Sjögren's syndrome and rheumatoid arthritis. Arthritis Rheum 1980;24:1274.

13. Mliyasaka N, Seaman W, Bakshi A, Sauvezie B, Strand V, Pope $R$. Talal N. Natural killing activity in Sjögren's syndrome. An analysis of the defective mechanisms. Arthritis Rheum 1983; 26:954. 14. Nagahiro JK. Ahira T, Shogo K, Fulmanaro $T$. Studies of the function of the natural killer-interferon systems in patients with Sjögren's syndrome. Clin Invest 1982;69:581.

15. Klimas NG, Salvato FR, Morgan R, Fletcher MA . Immunologic abnormalities in chronic fatigue syndrome. J Clin Microbiol 1990;28:1403-10.

16. Caldwell $C L$, Irwin $M$, Lohr $J$. Reduced natural killer cell cytotoxicity in depression but not in schizophrenia. Biol Psychiatry 1991:30:1131-8.

17. Locke SE, Kraus L, Leserman J, Hurst MW, Heisel JS, Williams M. Life change stress, Psychatric symptoms, and Natural killer cell activity. Psychosomatic Med 1984;46:441-53.
18. Schedlowski M, Jacobs R. Stratmann G, Richter S, Hädicke A, Tewes U, Wagner TOF, Schmidt RE. Changes of natural killer cells during acute psychological stress. J Clin Immunol 1993:13: 119-126.

19. Glaser R, Rice J, Speicher CE. Stont JC, Kiecolt-Glaser JK. Stress depresses interferon production by leukocytes concomitant with decrease in natural killer cell activity. Behav Neurosci 1986;100: 675-8.

20. Irwin M, Daniels M, Bloom E, Weiner H. Depression, and natural killer cell activity. Psychopharmacol Bull 1986;22: 1093-96.

21. Whiteside TL, Bryant J, Day R, Herberman RB. Natural killer cytotoxicity in the Diagnosis of immune dysfunction: Criteria for a reproducible assay. J Clin Lab Anal 1990;4:102-114.

22. Pross HF, Baines MG, Rubin P, Shragge P, Patterson MS. Spontaneous human lymphocyte-mediated cytotoxicity against tumor target cells. IX. The Quantitation of natural killer cell activity. J Clin Immunol 1981;1: 51-63.

23. Pross HF, Maroun JA. The standardization of NK cell assays for use in studies of biological response modifiers. J Immunol Meth 1984;68:235-49.

24. Baxevanis CN, Reclos G, Gritzapis AD, Dedousis GVZ, Missitzis I, Papamichail M. Elevated prostaglandin E2 production by monocytes is responsible for the depressed levels of natural killer and lymphokine-activated killer cell function in patients with breast cancer. Cancer 1993;72:491-501.

25. Cosentino LM, Cathart MK. A multi-step isolation scheme for obtaining $\mathrm{CD}_{16} 6^{+}$human natural killer cells. J Immunol Meth 1987:103,195-204. 
Hämatologie in der Praxis. 2. Auflage. H. Heimpel, D. Hoelzer, H.-P. Lohrmann, E. Seifried. Unter Mitarbeit von E. Kleihauer. Jena: Gustav Fischer Verlag, 1996, 372 pp, 21 Abbildungen im Text, 47 Farbabbildungen in Anhang, $24 \times 17 \mathrm{~cm}$, gebunden. 118,00 DM. \$73.00 öS. 113.50 sFr. ISBN 3-334-60815-8.

Die zweite, als völlig überarbeitet und erweitert angekündigte Auflage entspricht ausnahmsweise den Versprechungen. Sowohl die Bildqualität des schmalen Anhangs als auch die graphische Aufbereitung der Tabellen und Merksätze haben eine deutliche Verbesserung erfahren. Zwar fehlen die Randstichworte, die die 1. Auflage kennzeichneten, aber die Übersichtlichkeit hat darunter keineswegs gelitten. Klar ist die Gliederung der einzelnen Kapitel in die Abschnitte:

- Systematik und klin. Befund

- Diagnostik

- Stadieneinteilung

- Therapie

- Verlauf und Prognose

(incl. Therapie-Nebenwirkungen)

- Aufgaben der Praxis

Es werden die Themenkreise Anämien, Neoplasien der Hämopoese, wie chronisch myeloproliferative Syndrome (MPS), akute Leukämien, myelodysplastische Syndrome (MDS), Morbus Hodgkin, maligne Non-Hodgin-Lymphome (NHL), Plasmozytom sowie seltenere Erkrankungen des Makrophagensystems nach diesem Schema abgehandelt, wobei erfreulich ist, daß obsolete Methoden der Diagnostik und Therapie rigoros gestrichen wurden, dafür neuere Behandlungsmethoden mit Wachstumsfaktoren. KnochenmarkTransplantation etc., übersichtlich dargestellt, Eingang fanden. Auch der aktuellen Entwicklung mit automatisierter Blutbilderstellung wurde Rechnung getragen.

Der Buchumfang wurde dadurch nur unwesentlich erweitert, was der Lesbarkeit und Orientierung deutlich zugute kommt. Als Erleichterung zum Verständnis können auch die Abkürzungen und gebräuchlichen Werte (jeweils im Deckel plaziert) angesehen werden.

Ärgerlich sind einige Druckfehler, die im Falle der Anämiediagnostik (Tab. 7) zu Verständnisschwierigkeiten führen könnten. Hyperchrom-mikrozytär bei Vit. $B_{12}$-Mangel ist schlechterdings ein Unding. Ein Erratum sowie eine Verbesserung der nächsten Auflage wäre hier angebracht.

Das Buch, das sich vorwortgemäß überwiegend an Haus- und Kinderärzte wendet, ist darüber hinaus sicher auch für Laborärzte und -personal höchst interessant. die sich neben ihrer unmittelbaren Befundung für Therapieverlauf und Prognose, sprich das Schicksal der Patienten interessieren, für die sie diese Befunde erheben.

\section{Dr. Silke Heller, Berlin}

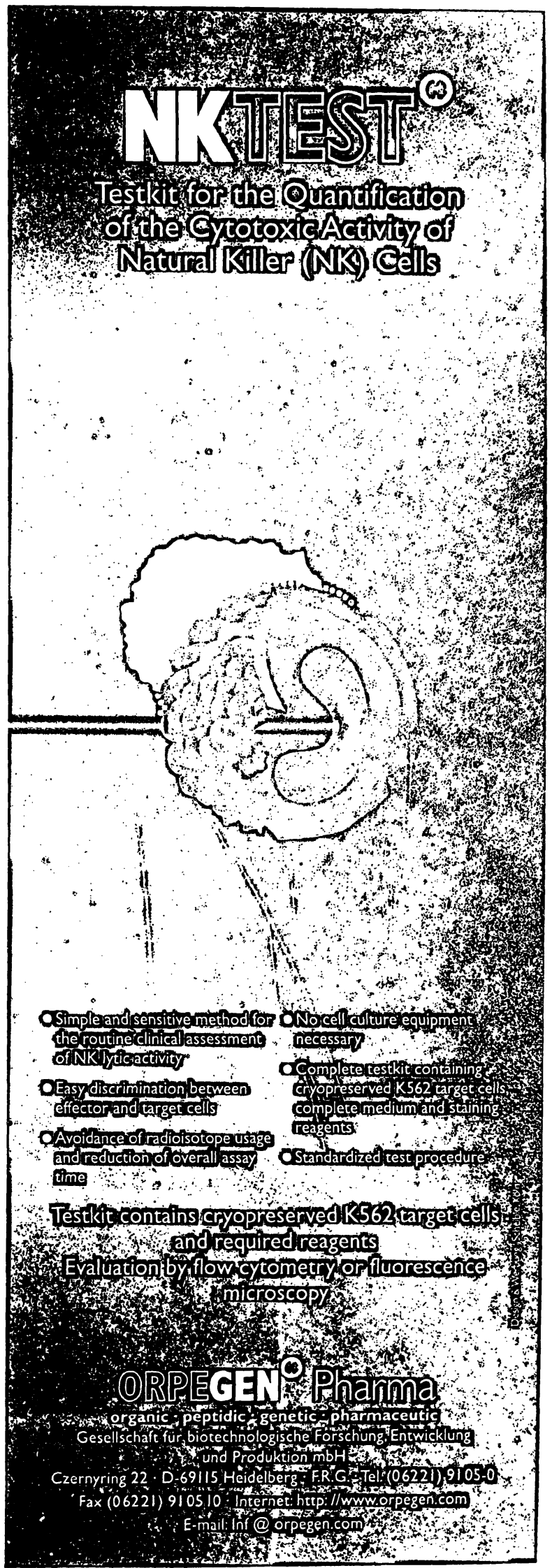

